\title{
BMJ Open Dutch multicentre, prospective follow-up, cohort study comparing the neurological and neuropsychological sequelae of hospitalised non-ICU- and ICU-treated COVID-19 survivors: a study protocol
}

\author{
Simona Klinkhammer (10 , 1,2 Janneke Horn, ${ }^{3,4}$ Johanna M A Visser-Meily, ${ }^{5,6}$ \\ Esmée Verwijk, ${ }^{7,8}$ Annelien Duits, ${ }^{1,2,9}$ Arjen J C Slooter, ${ }^{6,10}$ \\ Caroline M van Heugten, ${ }^{1,2,11}$ the NeNeSCo study group
}

To cite: Klinkhammer S, Horn J, Visser-Meily JMA, et al. Dutch multicentre, prospective follow-up, cohort study comparing the neurological and neuropsychological sequelae of hospitalised non-ICU- and ICU-treated COVID-19 survivors: a study protocol. BMJ Open 2021;11:e054901. doi:10.1136/ bmjopen-2021-054901

- Prepublication history for this paper is available online. To view these files, please visit the journal online (http://dx.doi org/10.1136/bmjopen-2021054901).

Received 02 July 2021 Accepted 19 September 2021

D) Check for updates

(c) Author(s) (or their employer(s)) 2021. Re-use permitted under CC BY-NC. No commercial re-use. See rights and permissions. Published by BMJ.

For numbered affiliations see end of article.

\section{Correspondence to}

Dr Caroline M van Heugten;

c.vanheugten@

maastrichtuniversity.nl

\section{ABSTRACT}

Introduction Owing to the novelty of COVID-19, there are still large knowledge gaps concerning its effect on the brain and the resulting impact on peoples' lives. This large-scale prospective follow-up study investigates COVID-19-associated brain damage, neuropsychological dysfunction and long-term impact on the well-being of patients and their close ones. It is hypothesised that structural brain damage and cognitive dysfunction primarily occur in severely ill patients, as compared with moderately ill patients. Cognitive complaints, emotional distress and impact on well-being are hypothesised to be less dependent on illness severity.

Methods and analysis For this multicentre study, 200 patients with COVID-19 (100 intensive care unit (ICU) patients and 100 non-ICU patients) formerly hospitalised in one of the six recruiting hospitals during the first European infection wave (ie, March to June 2020) and their close ones will be recruited. At minimally 6 months posthospital discharge, patients will perform a set of neuropsychological tests and are subjected to a $3 \mathrm{~T}$ MRI scan. Patients and close ones will fill out a set of questionnaires, also at minimally 6 months posthospital discharge and again another 6 months thereafter. Data related to COVID-19 hospitalisation will be extracted from the patients' medical records. MRI abnormalities will ultimately be related to neuropsychological test performance and questionnaire outcomes.

Ethics and dissemination Ethics approval was granted by the medical research ethics committee of Maastricht University Medical Centre and Maastricht University (NL75102.068.20). The project is sponsored by The Brain Foundation Netherlands. Findings will be presented at national and international conferences, as well as published in peer-reviewed scientific journals. Trial registration number NCT04745611.

\section{INTRODUCTION}

\section{Background and rationale}

COVID-19 has become known for its damage to the respiratory system. However, as the number

\section{Strengths and limitations of this study}

- This will be the first multicentre study to provide insight into the relationship between COVID-19associated brain damage, neuropsychological dysfunction, cognitive complaints and well-being in the long term.

- The sample selection allows a comparison of consequences in severely ill (intensive care unit (ICU)) and moderately ill (non-ICU) patients.

- The study results will benefit from enhanced generalisability and population representability due to the multicentre design.

- The study sample is limited to the COVID-19 survivors of the first infection wave in Dutch hospitals.

of infected individuals grew, so did the concern about the potential impact of this novel disease on the brain. Concerns were fuelled by knowledge of other coronaviruses affecting the brain and were strengthened by accumulating reports of neurological manifestations (eg, headaches, dizziness, loss of taste and smell). ${ }^{1-4}$ These manifestations demonstrate an impact of the disease on the central nervous system, with more severe manifestations occurring in more severely ill patients. ${ }^{1-3}$

The impact of COVID-19 on the brain was observed through MRI studies, which reported various forms of brain damage in this patient population. Among these were frequent reports of acute and subacute infarcts, microhaemorrhages, white matter hypodensities/ hyperintensities, leptomeningeal enhancement and cortical fluid-attenuated inversion recovery (FLAIR) signal abnormalities (induced by, eg, encephalitis, postictal state or ischaemia). ${ }^{56}$ Preliminary results suggest that, like neurological symptoms, brain damage 
also finds a stronger representation within more severely ill patients. ${ }^{23}$ The biological mechanisms proposed to contribute to COVID-19-related brain damage are viral invasion of the nervous system, hyperinflammation, vasculitis, hypoxia and hypercoagulation. ${ }^{7-9}$

The observed brain damage may result in a neuropsychological dysfunction. Retrospective research indeed suggests cognitive dysfunction (eg, memory, attention and executive dysfunction) and emotional distress (eg, anxiety and depression). ${ }^{10-12}$ However, the need was voiced for prospective long-term investigations to determine the prevalence, nature and extent of neuropsychological dysfunction. ${ }^{11}$ Furthermore, cognitive dysfunction may be associated with cognitive complaints. However, the mapping between cognitive dysfunction and cognitive complaints is not straightforward. Cognitive complaints are not always attributable to brain damage and cognitive dysfunction. Instead, they may also result from other factors, such as depression and fatigue, leading to an objective-subjective discrepancy. ${ }^{13}$ Furthermore, while patients may suffer from neurological and neuropsychological dysfunction, also their close ones (eg, relatives or friends) may be adversely affected. Close ones of severely ill patients are at high risk for experiencing emotional distress such as anxiety, post-traumatic stress and depression. ${ }^{14}$ This highlights the importance of adequate assessment of complaints and dysfunctions, which can ultimately guide the way for aftercare for survivors and close ones.

Although many studies investigate the impact of COVID-19 on physical and mental health separately, the interplay between brain damage, neuropsychological dysfunction and well-being has not yet been investigated. This comprehensive knowledge will facilitate post-COVID-19 care and may thereby reduce long-term negative disease impact. We expect neurological consequences and therefore cognitive dysfunction to mostly occur in more severely ill patients. In contrast, cognitive complaints, emotional distress and decreased well-being are hypothesised to be present in moderately ill patients as well.

\section{Study objectives}

This investigation answers the call for longitudinal prospective follow-up studies and aims to uncover COVID19-related neurological consequences and neuropsychological dysfunction comparatively for severely ill (ie, ICU) and moderately ill (ie, non-ICU) hospital-admitted patients. ${ }^{1115}$ The specific objectives of the study are as follows:

- Investigate the presence and nature of neurological consequences (ie, neurological symptoms and structural brain damage).

- Assess the presence and extent of neuropsychological dysfunction (ie, cognitive dysfunction and emotional distress).

- Relate cognitive complaints to neuropsychological dysfunction and underlying structural brain damage.

- Analyse the long-term impact of neurological consequences, neuropsychological dysfunction and cognitive complaints on the well-being of patients and their close ones.

- Identify clinical predictors associated with greater risk for structural brain damage and neuropsychological dysfunction.

\section{METHODS AND ANALYSIS}

\section{Study design}

The study is a multicentre prospective follow-up cohort study. At minimally 6 months posthospital discharge (ie, timepoint 1 (T1)), patients will (a) undergo an MRI brain scan to assess structural brain damage, (b) complete a neuropsychological test battery to examine cognitive dysfunction and (c) complete a set of questionnaires to investigate neurological symptoms, emotional distress and well-being. Another 6 months thereafter (ie, timepoint 2; T2), the set of questionnaires will be filled out a second time. Likewise, two sets of questionnaires, measuring emotional distress and well-being, will be completed by close ones at T1 and T2. (d) Data concerning hospital admission and disease characteristics will be extracted from the patients' medical files. To assess differences with regard to disease severity (ie, severely ill and moderately ill), measures will be compared between ICU-treated and non-ICU-treated patients.

\section{Participants}

The population consists of (a) patients with COVID-19 admitted to one of the six Dutch recruiting hospitals (ie, Amsterdam UMC, Maastricht UMC, UMC Utrecht, Zuyderland MC, Onze Lieve Vrouwe Gasthuis and Diakonessenhuis Utrecht) during the first European infection wave (March to June 2020) and (b) individuals who maintain regular and close contact with a participating patient, referred to as close ones.

\section{Inclusion criteria}

Patients with COVID-19

- Objectified SARS-CoV-2 infection for which ICU or non-ICU hospitalisation was necessary between March and June 2020.

- Admission to one of the six recruiting hospitals.

- 18 years or older.

- Mastering the Dutch language sufficiently to follow test instructions and answer questionnaires.

- Informed consent.

Close ones

- 18 years or older.

- Mastering the Dutch language sufficiently to follow test instructions and answer questionnaires.

- Informed consent.

\section{Exclusion criteria}

Patients with COVID-19

- Objectified cognitive impairment before hospital admission as confirmed by a treating physician.

- Severe structural neurological damage occurring after hospital discharge (ie, stroke and traumatic brain injury). 
- Physical inability to attend a hospital visit.

- Contraindications for MRI scanning (eg, metallic implants, implantable cardioverter defibrillators, claustrophobia and pregnancy).

\section{Sample size calculation}

We aim to include 100 ICU and 100 non-ICU survivors, plus one optional close one per patient. These numbers are based on expected group differences in cognitive dysfunction and grounded in group differences among similar populations. Normative values on a global measure of cognitive impairment (ie, Montreal Cognitive Assessment (MoCA)) were compared between a cohort of healthy elderly individuals and a mild stroke cohort, representing the non-ICU and ICU groups, respectively. ${ }^{1617}$ Based on that, an effect size of 0.44 was estimated for differences in cognitive dysfunction between the nonICU and ICU groups. As brain damage commonly manifests in cognitive dysfunction, we assume this estimated effect size to be similarly applicable to group differences in cognitive dysfunction. A power calculation with this effect size, power of $80 \%$ and two-sided $\alpha$ of 0.05 suggests a minimum of 81 participants per group. Taking dropout into account, the recruitment of 100 patients per group (ie, 200 patients in total) should result in sufficient statistical power to detect an effect of the estimated size. As the first measurement is executed during a hospital visit, we expect no dropouts at this stage. For the second measurement timepoint, we anticipate a dropout rate of $10 \%-15 \%$. To minimise attrition, participants will receive weekly reminders for a total of 4 weeks to fill in the second set of questionnaires unless they withdraw consent.

\section{Recruitment process}

A summary of the recruitment process is shown in figure 1. In each of the six recruiting hospitals, a treating physician will assess the eligibility of randomly selected patients based on hospital records of hospitalised patients. The

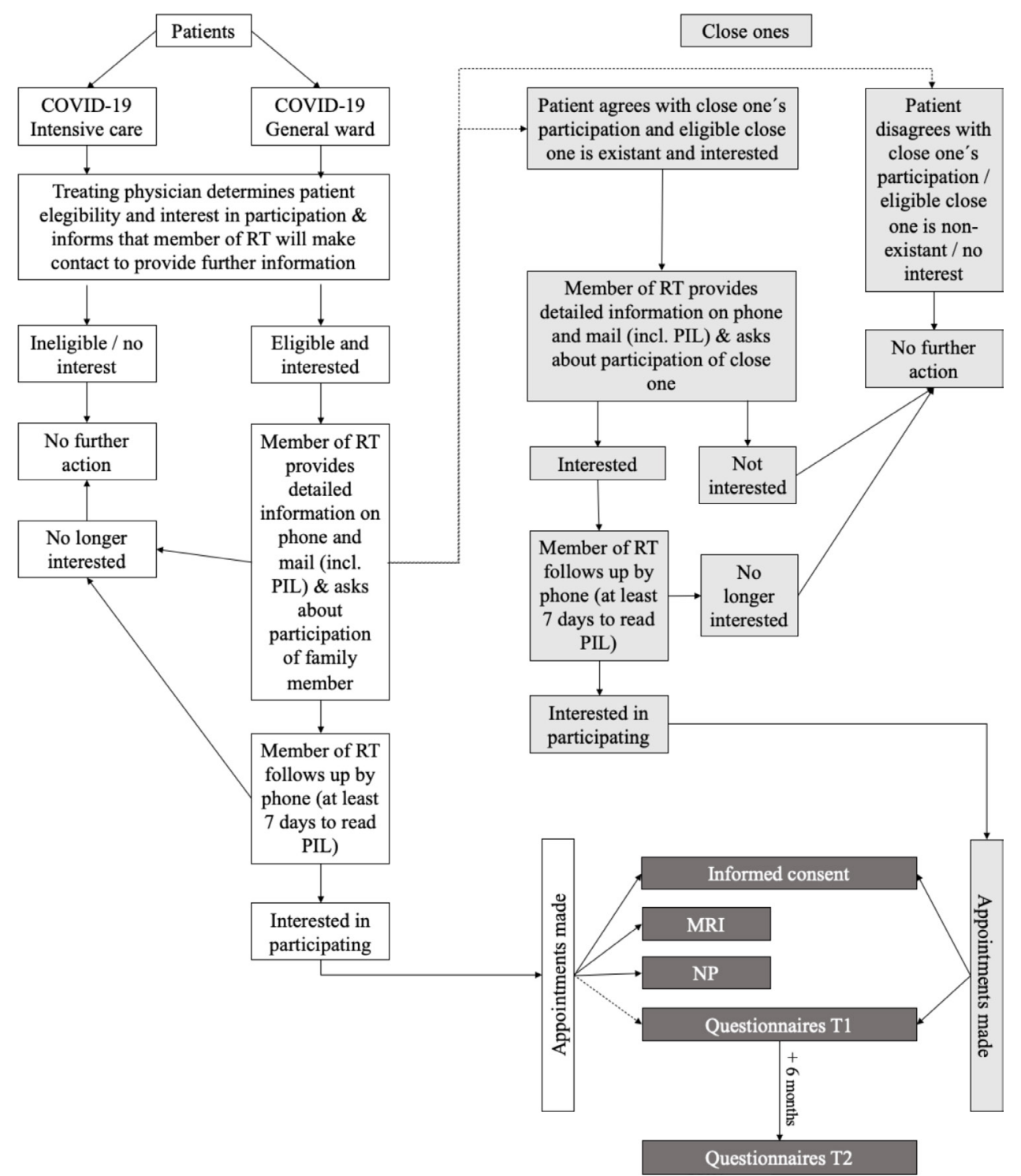

Figure 1 The recruitment process. MRI, magnetic resonance imaging; NP, neuropsychological testing; PIL, participant information letter; RT, research team; T1, timepoint 1; T2, timepoint 2. 
physician will then enquire whether eligible patients are interested in study participation. If a patient is interested, potential study participation will be discussed with a research assistant via telephone. The patient will be asked about the presence of a partner, family member or another individual close enough for being able to answer a detailed questionnaire about the patient's physiological and mental health before and after hospital admission. The close one should preferably have daily but at least weekly contact with the patient. In case a close one is available, and the patient agrees with joint participation, the close one will likewise be informed. Subsequently, information material will be sent to both patient and close one. Participation of a close one is not a requirement for patient participation in the study. Minimally 7 days following receipt of the information material, a second phone call will be made to determine continued participation interest, to answer questions and schedule the visit. In case of a participation-agreement, the patient and, where applicable, the close one, are invited for a hospital visit at one of the three participating university medical centres (ie, Amsterdam UMC, Maastricht UMC and UMC Utrecht).

\section{Study procedures}

The hospital visit will take place at T1. After having given informed consent, patients will receive (a) a structural MRI brain scan, specified below, performed at the local radiology departments according to standard procedures (including an MRI safety check) and (b) neuropsychological testing, carried out by trained research assistants. Meanwhile, close ones will fill out a set of questionnaires available digitally or on paper. Patients will likewise fill out a set of questionnaires (ie, questionnaires T1) with the option to do so on-site or at their home. If scanning and testing are not possible on the same day (eg, due to fatigue or hospital scheduling limitations), two separate appointments will be scheduled maximally 1 week apart. At T2, patients and close ones will again fill out the set of questionnaires (ie, questionnaires T2). Additionally, by signing the informed consent, patients grant the researchers access to medical file data collected during their COVID-19 hospital stay. MRI scans will be evaluated by an independent, certified neuroradiologist at one of the hospitals (ie, MUMC+). The scan evaluation will take place blinded with regard to the patients' prior hospitalisation and neuropsychological test performance. All data will be analysed by the executing researcher who will be blinded to data of the patients' hospital admission.

\section{Outcome measures and measurement tools MRI protocol}

Patients will undergo a 3T MRI scan to investigate structural brain damage. The scan protocol consists of the following sequences: T1-weighted, T2-weighted, FLAIR, susceptibility-weighted imaging and diffusion-weighted imaging. These sequences allow optimal evaluation of the brain damage expected based on the earlier discussed literature and proposed underlying mechanisms.

\section{Neuropsychological functioning}

A neuropsychological test battery will be administered to assess cognitive dysfunction (taking approximately 2 hours, including short breaks). This battery has been compiled by experts in the field and is based on current literature. ${ }^{18}$ These tests will be used to evaluate the following:

- Global cognitive function with the MoCA. ${ }^{19}$

- Memory with the Rey's auditory verbal learning test. ${ }^{20}$

- Executive function and attention with the Trail Making Test A/B, ${ }^{21}$ Stroop Test ${ }^{22}$ and Digit Span Test (subtest from the Wechsler Intelligence Scales ${ }^{23}$ ).

- Visuospatial function with Judgement of Line Orientation. ${ }^{24}$

- Language function with the Boston Naming Task, ${ }^{25}$ Controlled Oral Word Associations Task ${ }^{24}$ and Category fluency. ${ }^{26}$

- Processing speed with the Symbol Digit Substitution Test (subtest from the Wechsler Intelligence Scales). ${ }^{23}$

- Symptom validity with the Test of Memory Malingering. ${ }^{27}$

\section{Questionnaires}

A set of questionnaires will be filled out to investigate (premorbid) physical functioning, neurological symptoms, emotional distress (depression, anxiety and post-traumatic stress symptoms), cognitive complaints, personal factors (coping), environmental factors (social support, caregiver strain), sleep (quality, satisfaction, fatigue) and well-being (social participation and quality of life). Table 1 gives an overview of the investigated domains, respective assessment tools, the time point of investigation and whether they will be administered to patients, close ones or both.

\section{Demographics and disease-related characteristics}

Demographic data such as age, sex, education, marital status, living arrangement and status of employment will be collected from patients and close ones through a questionnaire at T1. Data that will be retrieved from the patient medical files concern (1) general hospital admission, such as length of stay, (2) disease-related parameters, such as the presence of coagulation disorders or renal failure and (3) treatment-related parameters, such as the need for mechanical ventilation, sedation and treatment with immunosuppressants. For this, we will make use of an existing national patient database (ie, CovidPredict). ${ }^{28}$

\section{Data analysis}

MRI analysis

Based on the findings of earlier studies and damage predicted by the formerly mentioned mechanisms, the analysis will evaluate the following aspects ${ }^{56}$ :

- White matter lesions by the use of Fazekas scores. ${ }^{29}$ 
Table 1 Outcome measures and measurement tools per time point and patients/close ones

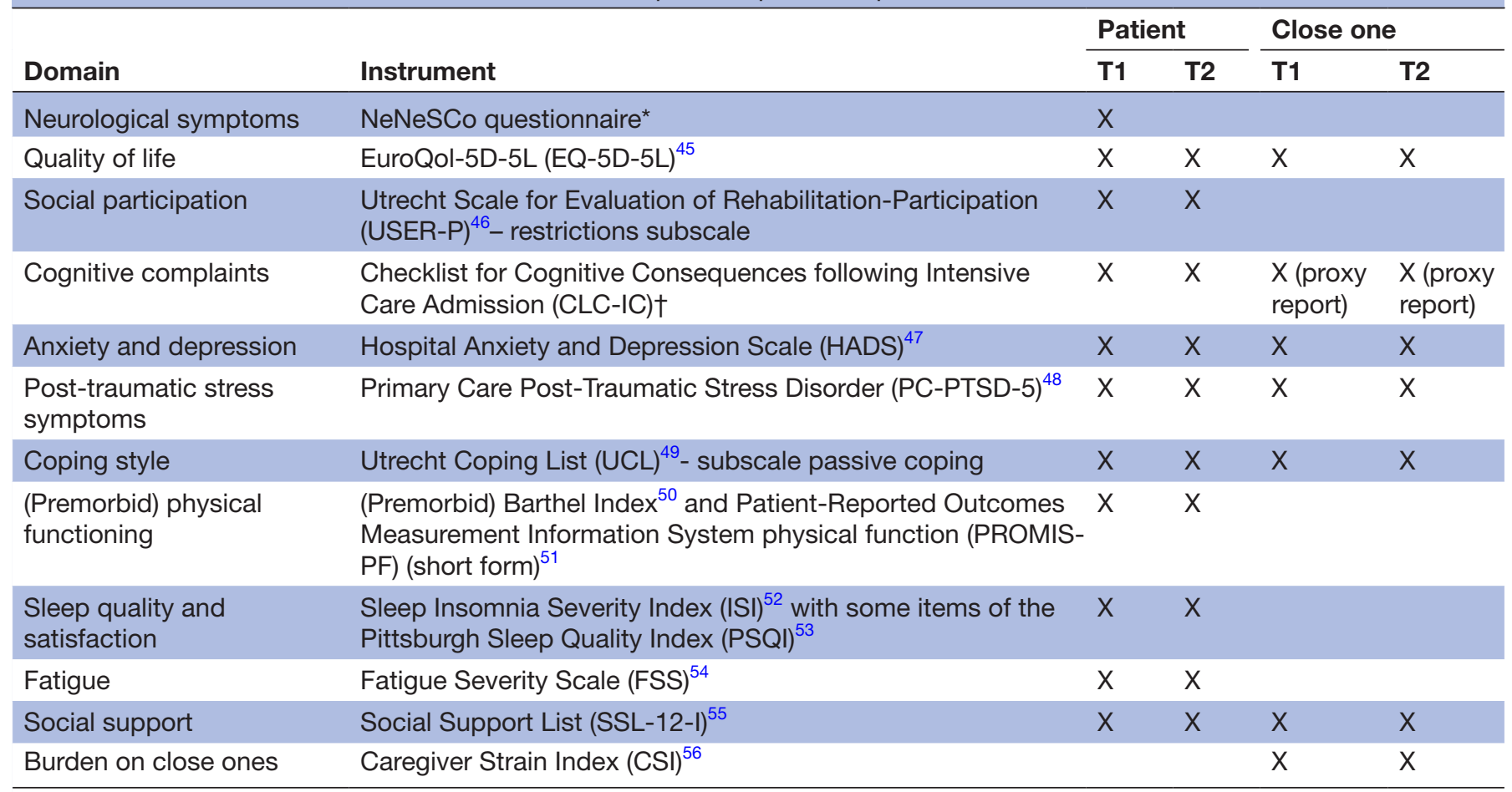

*Specifically designed for this study, assesses frequently recorded neurological symptoms associated with COVID-19 (ie, taste, smell, and vision, (neuropathic-) pain, headaches, tingling, or numbness in hands/feet and muscle weakness).

†The CLC-IC is an adapted version of the Checklist for Cognition and Emotion (CLCE-24). ${ }^{57}$

- Cerebral infarcts (specified by location: frontal/parietal/temporal/occipital/cerebellar and left hemisphere/right hemisphere).

- Lacunar infarcts (specified by location: basal ganglia/ cerebellar/lobar/brainstem).

- FLAIR hyperintensities other than white matter lesions and infarcts.

- Number of perivascular spaces (highest number in one hemisphere in a four-category scale; ${ }^{30}$ separately for basal ganglia and centrum semiovale).

- Haemorrhages (specified by location).

- Number of microhaemorrhages (in a three-category scale $;^{31}$ separately for lobar and basal ganglia).

- Global cortical atrophy through the use of the Pasquier scale. $^{29}$

- Medial temporal lobe atrophy through the use of the Scheltens' scale. ${ }^{29}$

The nature of the brain damage will be described per patient group and groups will be compared with regard to the presence of injury.

\section{Neuropsychological test battery analysis}

To evaluate cognitive dysfunction, participant scores on the various neuropsychological tests will be compared with age, sex and education corrected norm values. Subsequently, mean performance will be reported per instrument and group. Percentages of cognitive impairments will be reported per group based on the norm values. Group performance differences will be statistically evaluated.

\section{Questionnaire analysis}

Where available, questionnaire scores will be related to cut-off scores. Mean group scores and percentages above/below the cut-off scores will be reported per questionnaire, compared between groups and timepoints.

\section{Demographics}

Demographic data will be presented in form of mean scores and proportions, separately per group.

\section{Comprehensive analysis}

Brain damage will be related to cognitive dysfunction, which in turn will be related to emotional distress, cognitive complaints and well-being. Parameters predicting brain damage and cognitive dysfunction will be identified using hospital-related, disease-related and treatment-related data. The presence and severity of the consequences of COVID-19 (objectives 1 and 2) will be analysed using the appropriate descriptive statistics and group-comparison tests (ICU vs non-ICU). To relate subjective (ie, questionnaires) and objective functioning (ie, neuropsychological tests and MRI) we will use correlational and regression analyses (objective 3). Long-term impact will be determined using t-tests and multilevel analyses (objective 4). Predictor identification will be done with regression analyses (objective 5). 


\section{Patient and public involvement}

The study employs a user committee that consists of experience-experts (ie, patients with COVID-19 and representatives of patient organisations for brain injury and postintensive care syndrome (PICS)) and healthcare professionals. The committee assembles periodically to discuss the aim of the research, its feasibility, potential improvements and study progress. The healthcare professionals have different backgrounds (eg, neuropsychologists, intensivists and rehabilitation physicians).

\section{DISCUSSION}

Despite the rapidly growing body of evidence, relatively little is known about the lasting effects that COVID-19 may have on its survivors, specifically concerning the relation between brain damage, neuropsychological dysfunction and well-being. The available literature suggests a potentially persistent impact on many areas of patients' lives but calls for research to fill the knowledge gaps and supply data on long-term impact. ${ }^{19}$ This study answers this call for action and assesses the long-term neurological and neuropsychological sequelae of COVID-19 in formerly hospitalised patients and the impact on the wellbeing of patients and close ones.

An important strength of this investigation lies in connecting brain pathology to cognitive dysfunction and cognitive complaints. First, this allows a precise characterisation of COVID-19-related brain damage and its consequences. Second, it differentiates between individuals with cognitive dysfunction and individuals with cognitive complaints that cannot be objectified. Although it is important to facilitate patient care and rehabilitation for the former group, it is equally important to acknowledge and offer help to the latter. This is especially the case since both objective dysfunction and cognitive complaints can reduce well-being. ${ }^{32}$ This study is the first in taking this investigational approach to COVID-19.

Moreover, this study not only gives insight into the impact of COVID-19 on patients but also into the wellbeing of close ones. Close ones of severely ill patients may develop emotional distress which can decrease wellbeing and reduce the ability to engage in supportive and care-taking functions. ${ }^{14}$ The hospitalisation of a patient is often experienced as traumatic, especially when ICU admission is necessary. This experience may be further enhanced by the constant confrontation with COVID19-related deaths in the media and the uncertainty of potential long-term health consequences. Furthermore, contact with the patient is limited and sometimes even impossible due to strict isolation measures, while the usual social support is reduced for similar reasons. This leaves the patients' close ones particularly vulnerable. By including them in this research, we not only gain insight into their needs but also offer a potential second target for indirectly supporting COVID-19 survivors.

One shortcoming of this research is the lack of a nonCOVID-19 ICU control group (ie, individuals treated on the ICU for reasons other than COVID-19). It is hypothesised that neurological consequences and the resulting neuropsychological dysfunctions are mostly limited to ICU-treated-individuals, as opposed to non-ICU-treated individuals. However, critical illness and associated treatment are commonly paired with non-specific brain damage (eg, atrophy, white matter lesions and haemorrhages) as well as with cognitive dysfunction and emotional distress. ${ }^{33-37}$ This impact on health, function and well-being is commonly summarised under the term PICS. The brain damage, as well as underlying mechanisms, at least partially, overlap with those proposed to be involved in COVID-19-associated brain damage. ${ }^{38} 39$ To delineate disease-specific effects from effects caused by more general factors of severe illness, findings would need to be compared with those of patients treated in the ICU without COVID-19, with similar disease severity and treatments. Data, including MRI and neuropsychological test data, for such cohorts, are available. ${ }^{40} 41$ The neuropsychological test battery used for this research consists of tests commonly used for research purposes and is therefore well-suited for later comparison with historical cohorts to delineate specific effects of COVID-19 from the more general effects associated with critical illness and ICU treatment.

A second shortcoming concerns a change in treatment, as COVID-19 treatment is steadily improving. Improvement comes, for example, from the administration of anti-inflammatory agents such as tocilizumab and dexamethasone. These agents were found to reduce mortality rates among severely ill patients with COVID-19, decrease the percentage of ICU admissions, reduce the need for mechanical ventilation and shorten the length of hospital stay. ${ }^{42-44}$ It is therefore also conceivable that the change of treatment will have led to different or reduced neurological and neuropsychological consequences. The chosen study sample is limited to the first infection wave, which potentially reduces the generalisability of results. One mechanism assumed to contribute to greater illness severity and COVID-19-associated brain damage is an excessive immune response. ${ }^{1}$ The reduction of the inflammatory response therefore reduces illness severity and thereby the risk of brain damage directly and indirectly (eg, by decreasing the need for mechanical ventilation). However, while the change in treatment decreases the risk, it likely does not eliminate it. Furthermore, other mechanisms may not even be affected by this change of medication. Nevertheless, to tackle this problem, the authors plan to extend the scope of this investigation and additionally include a group of patients from the second and third European COVID-19 wave in the future.

ICU and non-ICU patients differ in illness severity and may differ as well regarding other characteristics, such as age. These potentially confounding effects will be assessed before executing the main analyses and will, if necessary, be controlled for. At last, study results may be subject to self-selection bias, whereby individuals with certain psychological complaints (eg, post-traumatic 
stress) refrain from study participation. Likewise, persons without complaints may decline participation, despite our efforts in clarifying that complaints are not a requirement for participation. To be able to estimate the influence of such factors, reasons for refraining from participation will be noted in detail.

Despite some methodological caveats, this investigation is among the first providing detailed insight into the complex relationship between COVID-19-associated brain damage and its consequences on neuropsychological dysfunction and cognitive complaints. The benefits clearly outweigh the limitations as patients as well as rehabilitation professionals are searching for answers and adequate aftercare. The findings of this study will be a first step in the right direction by supplying the necessary grounds that future care and research can build on.

\section{ETHICS AND DISSEMINATION}

Ethics approval for the study has been obtained from the medical research ethics committee of Maastricht University Medical Centre and Maastricht University (committee reference number NL75102.068.20) and from the local committees of participating clinical centres. Participants will receive written and verbal information about the nature and aim of the study before giving consent. Participants are informed that participation is voluntary and about their right to withdraw at any time and without giving reasons. The handling of personal data will comply with the General Data Protection Rule and the Research Data Management Code of Conduct of Maastricht University. In accordance with the Central Committee on Research Involving Human Subjects statement on publication policy, the researchers aim to publish the study results (positive or negative) in international, highimpact peer-reviewed scientific journals. Furthermore, results will be presented at professional conferences and provided to study participants.

\section{Author affiliations}

${ }^{1}$ Department of Psychiatry and Neuropsychology, Maastricht University Faculty of Health Medicine and Life Sciences, Maastricht, The Netherlands

${ }^{2}$ School for Mental Health and Neuroscience, Maastricht University, Maastricht, The Netherlands

${ }^{3}$ Department of Intensive Care, Amsterdam UMC Locatie AMC, Amsterdam, The Netherlands

${ }^{4}$ Amsterdam Neuroscience, Amsterdam UMC Locatie AMC, Amsterdam, The Netherlands

${ }^{5}$ Department of Rehabilitation, Physical Therapy Science and Sports, UMC Utrecht, Utrecht, The Netherlands

${ }^{6}$ UMC Utrecht Brain Center, UMC Utrecht, Utrecht, The Netherlands

${ }^{7}$ Department of Medical Psychology, Amsterdam UMC Locatie AMC, Amsterdam,

The Netherlands

${ }^{8}$ Department of Psychology, Brain and Cognition, University of Amsterdam, Amsterdam, The Netherlands

${ }^{9}$ Department of Medical Psychology, Maastricht UMC+, Maastricht, The Netherlands ${ }^{10}$ Department of Intensive Care, UMC Utrecht, Utrecht, The Netherlands

${ }^{11}$ Department of Neuropsychology and Psychopharmacology, Faculty of Psychology and Neuroscience, Maastricht University, Maastricht, The Netherlands
Correction notice This article has been corrected since it was published. Name of Professor Johanna M A Visser-Meily has been spelled correctly.

Collaborators The following are members of the NeNeSCo (Neurological and Neuropsychological Sequelae of COVID-19) study group: Marcel J.H. Aries, Bas C.T. van Bussel, Jaap F.A. Jansen, Mark L.F. Janssen, Alida A. Postma, Susanne van Santen, Fabienne J.H. Magdelijns, Rein Posthuma, Meta van der Woude, Amy Otten, Attila Karakus, Inez Bronsveld, Niek Galenkamp, Karin A.H. Kaasjager, Dook W. Koch, Gert J. Geurtsen, Matthijs C. Brouwer, Kees Brinkman and Wytske A. Kylstra.

Contributors Study design: $\mathrm{CMvH}$ and JH; Development of the study protocol: CMvH, JH, SK, JMAV-M, AD, EV and AJCS; Patient recruitment: SK; Data collection: SK; Manuscript preparation: CMvH, JH, SK, JMAV-M, AD, EV and AJCS. The members of the NeNeSCo study group co-designed the study protocol, selected potential participants, assisted in their recruitment, collected data, and set up, prepared, and hosted COVID-19 databases. All authors read and approved the final manuscript.

Funding This study was supported by The Brain Foundation Netherlands grant number DR-2020-00377.

Competing interests None declared.

Patient and public involvement Patients and/or the public were involved in the design, or conduct, or reporting, or dissemination plans of this research. Refer to the Methods section for further details.

Patient consent for publication Not applicable.

Provenance and peer review Not commissioned; externally peer reviewed.

Open access This is an open access article distributed in accordance with the Creative Commons Attribution Non Commercial (CC BY-NC 4.0) license, which permits others to distribute, remix, adapt, build upon this work non-commercially, and license their derivative works on different terms, provided the original work is properly cited, appropriate credit is given, any changes made indicated, and the use is non-commercial. See: http://creativecommons.org/licenses/by-nc/4.0/.

ORCID iD

Simona Klinkhammer http://orcid.org/0000-0001-7008-0323

\section{REFERENCES}

1 Leonardi M, Padovani A, McArthur JC. Neurological manifestations associated with COVID-19: a review and a call for action. J Neurol 2020;267:1573-6.

2 Kremer S, Lersy F, de Sèze J, et al. Brain MRI findings in severe COVID-19: a retrospective observational study. Radiology 2020;297:E242-51.

3 Kandemirli SG, Dogan L, Sarikaya ZT, et al. Brain MRI findings in patients in the intensive care unit with COVID-19 infection. Radiology 2020;297:E232-5.

4 Correia AO, Feitosa PWG, Moreira JLdeS, et al. Neurological manifestations of COVID-19 and other coronaviruses: a systematic review. Neurology, Psychiatry and Brain Research 2020;37:27-32.

5 Egbert AR, Cankurtaran S, Karpiak S. Brain abnormalities in COVID-19 acute/subacute phase: a rapid systematic review. Brain Behav Immun 2020;89:543-54.

6 Gulko E, Oleksk ML, Gomes W, et al. Mri brain findings in 126 patients with COVID-19: initial observations from a descriptive literature review. AJNR Am J Neuroradiol 2020;41:2199-203.

7 Pezzini A, Padovani A. Lifting the mask on neurological manifestations of COVID-19. Nat Rev Neurol 2020;16:636-44.

8 Mazeraud A, Righy C, Bouchereau E, et al. Septic-Associated encephalopathy: a comprehensive review. Neurotherapeutics 2020;17:392-403.

9 Carod-Artal FJ. Neurological complications of coronavirus and COVID-19. Rev Neurol 2020;70:311-22.

10 Almeria M, Cejudo JC, Sotoca J, et al. Cognitive profile following COVID-19 infection: clinical predictors leading to neuropsychological impairment. Brain Behav Immun Health 2020;9:100163.

11 Jaywant A, Vanderlind WM, Alexopoulos GS. Frequency and profile of objective cognitive deficits in hospitalized patients recovering from COVID-19. Neuropsychopharmacology 2021:1-6.

12 Taquet M, Luciano S, Geddes JR, et al. Bidirectional associations between COVID-19 and psychiatric disorder: retrospective cohort studies of 62354 COVID-19 cases in the USA. Lancet Psychiatry 2021;8:130-40.

13 Vos L, Williams MW, Poritz JMP, et al. The discrepancy between cognitive complaints and neuropsychological test findings in persons with traumatic brain injury. J Head Trauma Rehabil 2020;35:E382-92. 
14 Davidson JE, Jones C, Bienvenu OJ. Family response to critical illness: postintensive care syndrome-family. Crit Care Med 2012;40:618-24.

15 Paterson RW, Brown RL, Benjamin L, et al. The emerging spectrum of COVID-19 neurology: clinical, radiological and laboratory findings. Brain 2020;143:3104-3120.

16 Borland E, Nägga K, Nilsson PM, et al. The Montreal cognitive assessment: normative data from a large Swedish population-based cohort. J Alzheimers Dis 2017;59:893-901.

17 Nijsse B, Visser-Meily JMA, van Mierlo ML, et al. Temporal evolution of poststroke cognitive impairment using the Montreal cognitive assessment. Stroke 2017;48:98-104.

18 Verwijk E, Geurtsen G, Renssen J. Aanbevelingen voor Het monitoren van cognitieve gevolgen bij post-IC COVID-19 patiënten, 2020. Available: https://www.Ivmp.nl/wp-content/uploads/2020/05/ Monitoring-COVID-19-patienten.vs2_.1.01052020-1.pdf [Accessed 19 May 2021].

19 Nasreddine ZS, Phillips NA, Bédirian V, et al. The Montreal cognitive assessment, MoCA: a brief screening tool for mild cognitive impairment. J Am Geriatr Soc 2005;53:695-9.

20 Rey A. L'examen clinique en psychologie, 1958.

21 Reitan RM, Wolfson D. The Halstead-Reitan neuropsychological test battery: theory and clinical interpretation: Reitan neuropsychology, 1985.

22 Stroop JR. Studies of interference in serial verbal reactions. J Exp Psychol 1935;18:643-62.

23 Wechsler D. Wechsler adult intelligence scale, 1955.

24 Benton AL, Abigail B, Sivan AB. Contributions to neuropsychological assessment: a clinical manual. USA: Oxford University Press, 1994.

25 Roth C. Boston Naming Test. In: Kreutzer JS, DeLuca J, Caplan B, eds. Encyclopedia of clinical neuropsychology. New York, NY: Springer New York, 2011: 430-3.

26 Benton AL. Differential behavioral effects in frontal lobe disease. Neuropsychologia 1968;6:53-60.

27 Iverson GL. Test of Memory Malingering. In: Kreutzer JS, DeLuca J, Caplan B, eds. Encyclopedia of clinical neuropsychology. New York, NY: Springer New York, 2011: 2494-6.

28 Ottenhoff MC, Ramos LL, Potters W. Predicting mortality of individual COVID-19 patients: a multicenter Dutch cohort. medRxiv 2020.

29 Wahlund L-O, Westman E, van Westen D, et al. Imaging biomarkers of dementia: recommended visual rating scales with teaching cases. Insights Imaging 2017;8:79-90.

30 Klarenbeek P, van Oostenbrugge RJ, Rouhl RPW, et al. Ambulatory blood pressure in patients with lacunar stroke: association with total MRI burden of cerebral small vessel disease. Stroke 2013;44:2995-9

31 Wilson D, Ambler G, Lee K-J, et al. Cerebral microbleeds and stroke risk after ischaemic stroke or transient ischaemic attack: a pooled analysis of individual patient data from cohort studies. Lancet Neurol 2019;18:653-65.

32 Hill NL, McDermott C, Mogle J, et al. Subjective cognitive impairment and quality of life: a systematic review. Int Psychogeriatr 2017;29:1965

33 Hopkins RO, Jackson JC. Long-Term neurocognitive function after critical illness. Chest 2006;130:869-78.

34 Suchyta MR, Jephson A, Hopkins RO. Neurologic changes during critical illness: brain imaging findings and neurobehavioral outcomes. Brain Imaging Behav 2010;4:22-34.

35 Jackson JC, Ely EW. Cognitive impairment after critical illness: etiologies, risk factors, and future directions. Semin Respir Crit Care Med 2013;34:216-22.

36 Hopkins RO, Suchyta MR, Beene K, et al. Critical illness acquired brain injury: neuroimaging and implications for rehabilitation. Rehabil Psychol 2016;61:151.
37 Brown $\mathrm{CH}$, Sharrett AR, Coresh J, et al. Association of hospitalization with long-term cognitive and brain MRI changes in the ARIC cohort. Neurology 2015;84:1443-53.

38 Al-Sarraj S, Troakes C, Hanley B, et al. Invited review: the spectrum of neuropathology in COVID-19. Neuropathol Appl Neurobiol 2021;47:3-16.

39 Lane-Fall MB, Kuza CM, Fakhry S, et al. The lifetime effects of injury: postintensive care syndrome and posttraumatic stress disorder. Anesthesiol Clin 2019;37:135-50.

40 Pandharipande PP, Girard TD, Jackson JC, et al. LongTerm cognitive impairment after critical illness. N Engl J Med 2013;369:1306-16.

41 Honarmand K, Lalli RS, Priestap F, et al. Natural history of cognitive impairment in critical illness survivors. A systematic review. Am J Respir Crit Care Med 2020;202:193-201.

42 Wise J. Covid-19: arthritis drug tocilizumab reduces deaths in hospitalised patients, study shows. BMJ 2021;372:n433.

43 Karagiannidis C, Windisch W, McAuley DF, et al. Major differences in ICU admissions during the first and second COVID-19 wave in Germany. Lancet Respir Med 2021;9:e47-8.

44 Ahmed $\mathrm{MH}$, Hassan A. Dexamethasone for the treatment of coronavirus disease (COVID-19): a review. SN Comprehensive Clinical Medicine 2020;2:2637-46.

45 Herdman M, Gudex C, Lloyd A, et al. Development and preliminary testing of the new five-level version of EQ-5D (EQ-5D-5L). Qual Life Res 2011;20:1727-36.

46 van der Zee $\mathrm{CH}$, Visser-Meily JMA, Lindeman E, et al. Participation in the chronic phase of stroke. Top Stroke Rehabil 2013;20:52-61.

47 Zigmond AS, Snaith RP. The hospital anxiety and depression scale. Acta Psychiatr Scand 1983;67:361-70.

48 Prins A, Ouimette P, Kimerling R, et al. The primary care PTSD screen (PC-PTSD): development and operating characteristics. Primary Care Psychiatry 2004:9:9-14.

49 Schreurs P, Van de Willige G, Brosschot J, et al. Handleiding utrechtse coping lijst UCL (herziene versie), 1993. Available: https:// bsw.ugent.be/VVGP/UCL.pdf [Accessed 19 May 2021].

50 Mahoney FI, Barthel DW. Functional evaluation: the Barthel index: a simple index of independence useful in scoring improvement in the rehabilitation of the chronically ill. Md State Med J 1965

51 U.S. Department of Health and Human Services. Patient-Reported outcomes measurement information system, 2021. Available: http:// www.nihpromis.org2021

52 Bastien $\mathrm{CH}$, Vallières $\mathrm{A}$, Morin $\mathrm{CM}$. Validation of the insomnia severity index as an outcome measure for insomnia research. Sleep Med 2001;2:297-307.

53 Buysse DJ, Reynolds CF, Monk TH, et al. The Pittsburgh sleep quality index: a new instrument for psychiatric practice and research. Psychiatry Res 1989;28:193-213.

54 Shirley Ryan Ability Lab. Fatigue severity scale 2016. Available: https://www.sralab.org/rehabilitation-measures/fatigue-severity-scale [Accessed 25 Mar 2021]

55 Kempen GIJM, Van Eijk LM. The psychometric properties of the SSL12-I, a short scale for measuring social support in the elderly. Soc Indic Res 1995;35:303-12.

56 Sullivan MT. Caregiver strain index (Csl). Home Healthc Nurse 2003;21:197-8

57 van Heugten C, Rasquin S, Winkens I, et al. Checklist for cognitive and emotional consequences following stroke (CLCE-24): development, usability and quality of the self-report version. Clin Neurol Neurosurg 2007;109:257-62. 